\title{
Phase-field study for the splitting mechanism of coherent misfitting precipitates in anisotropic elastic media
}

\author{
Pil-Ryung Cha * Dong-Hee Yeon, and Jong-Kyu Yoon \\ School of Materials Science and Engineering, Seoul National University, Seoul, Korea 151-742.
}

(Dated: June 8, 2018)

\begin{abstract}
Coherent misfitting precipitates in elastically stressed media such as $\gamma^{\prime}$ particles in nickel-based super-alloys show various splitting patterns such as doublets, quartets, or octets due to their misfit strain energy. While it is an interesting instability phenomenon defying conventional surface thermodynamics, its mechanism is not completely clear. Through a phase-field study upon the splitting behavior and morphological evolution of coherent precipitates, we show that an interface instability driven by elastic anisotropy and a diffusion field can generate elastically induced splitting during diffusional phase transition. Particle splitting is triggered by interface grooving which advances by penetrating grooves into the interior of the particle. The sequential evolution of shapes during the splitting process is in good agreement with previous experiments.
\end{abstract}

PACS numbers: $64.70 . \mathrm{Kb}, 81.30 .-\mathrm{t}, 81.40 . \mathrm{Cd}$

Keywords:

Elastic stresses arising during solid state phase transitions in some alloys can lead to microstructural features such as precipitate alignment, cuboidal precipitate shapes, and particle splitting. A wealth of experimental and theoretical works have reported how the mismatch strain between the precipitate and the surrounding matrix phase changes the characteristics of the microstruc-

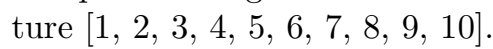

One of the primary reasons for considering the role of elasticity in microstructure formation is that it offers us the possibility of using elastic stresses to design desirable microstructures. In this context, one of the possibilities often cited is that of inverse coarsening. In inverse coarsening, the elastic fields promote the growth of a small precipitate at the expense of a larger neighbor - exactly the opposite to surface energy driven coarsening. In principle, elasticity could favor an array of equally sized precipitates, which would lead to the improved mechanical properties of the alloy.

Particle splitting is perhaps the most closely linked elastic effect to inverse coarsening. Particle splitting is a process in which one cuboidal particle splits into several particles, usually two (a doublet) or eight (an octet). Such splitting has been observed experimentally 2, 3] and has been theoretically identified as an elastically driven process [5, 6].

While splitting may be an important factor for inverse coarsening, its mechanism and the precise experimental and theoretical conditions under which it occurs are not completely clear. Some experimental pictures suggest that splitting occurs via a morphological instability in which the sides of a cuboidal precipitate become increasingly concave prior to splitting [3]. Other pictures suggest a hollowing at the center of the particle [5]. Many theoretical simulations of splitting have used diffuse interface techniques, and suggest that the instability occurs by the 'hollowing' mechanism [7, 8]. However, the hollowing mechanism cannot explain the formation of an octet and the fact that the splitting occurs mostly in the concave particle shape [2, 3] though it can describe splitting to a doublet. Recently, Lee, using a discrete atom method, observed splitting by an instability mechanism [9].

In this letter we demonstrate, through phase field model calculations, that interface instability driven by elastic anisotropy and diffusion field can generate particle splitting. Splitting occurred only under the concave growth condition and the picture that a concentrated elastic field around interface groove promotes splitting is very similar to Asaro-Tiller-Grinfeld (ATG) instability 11]. In addition, this splitting mechanism can explain the formation of both doublets and octets.

The model in this study assumes coherent interfaces (i.e. there are no dislocations) and takes into account the effect of anisotropic elasticity and elastic inhomogeneity which represents that the elastic constants of the matrix and the precipitate differ. We assume that the misfit between the matrix and the precipitate is purely dilatational with magnitude $e^{T}$, the elastic constants of both phases have cubic (four-fold) symmetry, and that the lattice parameter is not a function of composition. The model is formulated in terms of the concentration field $c(\vec{r}, t)$, the phase field $\phi(\vec{r}, t)$ which is zero in the matrix phase and one in the precipitate, and the elastic strain tensor $u_{i j}=1 / 2\left(\partial u_{i} / \partial x_{j}+\partial u_{j} / \partial x_{i}\right)$, where $\vec{u}(\vec{r}, t)$ is the atomic displacement field. The interfacial region is defined to be a mixture of matrix and precipitate with a different composition but with the same chemical potential [13]. Therefore, the concentration field of the system is defined as $c=c^{P} h(\phi)+c^{M}[1-h(\phi)]$, where $c^{A}$ is a concentration in the A-phase and $h(\phi)$ is the smooth interpolation function, which is zero at $\phi=0$ and one at $\phi=1$ and changes smoothly from zero to one, i.e. $0<\phi<1$. The superscript $P$ and $M$ represent the pre- 
cipitate and matrix phases, respectively, which will be used later without description. From the definition of the interfacial region, the following constraint should be satisfied at any point in the system:

$$
f_{c^{P}}^{P}\left(c^{P}\right)=f_{c^{M}}^{M}\left(c^{M}\right)
$$

where $f^{A}$ is the chemical free energy of the A-phase and $f_{c^{A}}^{A}$ represents the derivative of $f^{A}$ with respect to $c^{A}$. The free energy functional is:

$$
\mathcal{F}=\int_{\vec{r}}\left[f\left(\phi, c, u_{i j}\right)+\frac{\varepsilon^{2}}{2}|\nabla \phi|^{2}\right] d \vec{r}
$$

where $f\left(\phi, c, u_{i j}\right)$ is the free energy density of the system including the coherent strain energy, defined as $w g(\phi)+$ $f^{P}\left(c^{P}\right) h(\phi)+f^{M}\left(c^{M}\right)[1-h(\phi)]+f^{e l}\left(\phi, u_{i j}\right)$, where $g(\phi)$ is the double well potential, which has minima at 1 and 0 , the equilibrium values of the precipitates and matrix phases,respectively. In this study, $g(\phi)$ was selected as $\phi^{2}(1-\phi)^{2}$. $f^{e l}$ is the coherent elastic energy density caused by the lattice mismatch between two phases. In general, the elastic energy density is expressed as [14]:

$$
f^{e l}=\frac{1}{2} C_{i j k l}(\phi)\left[u_{i j}-e^{T} \delta_{i j} \psi(\phi)\right]\left[u_{k l}-e^{T} \delta_{k l} \psi(\phi)\right]
$$

where $C_{i j k l}$ is the stiffness tensor that depends on the phase field due to elastic inhomogeneity and $\psi(\phi)$ is a smooth function with the same characteristics as $h(\phi)$. A summation convention over repeated indices is implicit. Considering the spatial inhomogeneity of the elastic stiffness, the stiffness tensor is given by:

$$
C_{i j k l}(\phi)=C_{i j k l}^{0}+C_{i j k l}^{\prime} \xi(\phi)
$$

where $\xi(\phi)$ is a smooth function which is zero at $\phi=1$ and one at $\phi=0, C_{i j k l}^{0}=C_{i j k l}^{P}$, and $C_{i j k l}^{\prime}=C_{i j k l}^{M}-C_{i j k l}^{P}$, where $C_{i j k l}^{A}$ is the elastic stiffness of the A-phase.

It is reasonable to suppose that the elastic field relaxes much faster than $c$ or $\phi$. The elastic field can then be solved in terms of the phase field using the condition of local mechanical equilibrium:

$$
\frac{\delta \mathcal{F}}{\delta u_{i}}=\nabla_{j} \sigma_{i j}=0
$$

where $\sigma_{i j}=\delta \mathcal{F} / \delta u_{i j}$ is the stress tensor:

$$
\sigma_{i j}=C_{i j k l}(\phi)\left[u_{k l}-e^{T} \delta_{k l} \psi(\phi)\right]
$$

In this work, in order to obtain accurate solutions of equation (5) with minimal calculation time, the high order approximation method similar to the method proposed by Chen and coworkers 15 is used instead of the iteration method which gives very accurate solutions but requires considerable calculation time.

The solution of equation (5) to zeroth order in the elastic stiffness $C_{i j k l}^{\prime}$ is

$$
\hat{u}_{k}^{0}=i \alpha_{k i}^{-1} \sigma_{i j}^{0} q_{j} \hat{\psi}(\hat{\phi})
$$

where $\hat{A}$ represents the value of $A$ in Fourier space, $q_{j}$ the $j$-direction component of the wave number vector, $\sigma_{i j}^{0}=C_{i j k l}^{0} e^{T} \delta_{k l}$, and $\alpha_{k i}^{-1}$, indicates a component of the inverse tensor of $\alpha_{i k}$ which is defined as $C_{i j k l}^{0} q_{j} q_{l}$. Then, the displacement field to the $n$th order becomes

$$
\begin{aligned}
\hat{u}_{k}^{n}=\hat{u}_{k}^{0} & +i \alpha_{k i}^{-1} C_{i j l m}^{\prime} e^{T} \delta_{l m} q_{j}\{\xi(\phi) \psi(\phi)\}_{F} \\
& -i \alpha_{k i}^{-1} C_{i j l m}^{\prime} q_{j}\left\{\left[\xi(\phi) \nabla_{m}\right] u_{l}^{n-1}\right\}_{F}
\end{aligned}
$$

where $u_{k}^{n-1}$ is the displacement field to the $n$ - 1 th order and \{\}$_{F}$ represent the Fourier transform of the term in braces.

The elastic field can now be expressed in terms of the phase field $\phi$. Substituting the solution for the strain field gives the free energy in terms of $c$ and $\phi$. Assuming that the system evolves in time so that its total free energy decreases monotonically, the evolution equation for the phase field becomes:

$$
\frac{\partial \phi}{\partial t}=-M \frac{\delta \mathcal{F}}{\delta \phi}=M\left(\varepsilon^{2} \nabla^{2} \phi-f_{\phi}\right),
$$

with

$$
f_{\phi}=h^{\prime}(\phi)\left\{f^{P}-f^{M}+\left(c^{M}-c^{P}\right) f_{c^{M}}^{M}\right\}+f_{\phi}^{e l}+w g^{\prime}(\phi)
$$

while the concentration field should evolve with:

$$
\begin{aligned}
\frac{\partial c_{k}}{\partial t} & =\nabla \cdot \frac{D(\phi)}{f_{c c}} \nabla \frac{\delta \mathcal{F}}{\delta c} \\
& =\nabla \cdot D(\phi) \nabla c+\nabla \cdot \frac{D(\phi)}{f_{c c}} f_{c \phi} \nabla \phi
\end{aligned}
$$

where $M$ is the phase-field mobility, $D(\phi)$ is the diffusivity of solute atoms, and $f_{\phi}, f_{\phi}^{e l}, f_{c c}$, and $f_{c \phi}$ are $\partial f / \partial \phi$, $\partial f^{e l} / \partial \phi, \partial^{2} f / \partial c^{2}$, and $\partial^{2} f / \partial c \partial \phi$, respectively.

In the sharp interface limit, the phase field model presented here reduces to a modified Gibbs-Thompson equation with a coherent interface as follows:

$$
\alpha \sigma v_{n}=\kappa_{c} \sigma+\Delta \mu_{C}+G^{c o h}+G^{\text {trans }}
$$

Here, $\alpha=D / M \varepsilon^{2}, v_{n}$ is the interface velocity, $\sigma$ the interface energy, $\kappa_{c}$ the curvature of the interface, and $\Delta \mu_{C}$ the chemical driving force of the phase transition defined as $\mu_{C}^{P}-\mu_{C}^{M}$, where $\mu_{C}^{A}$ represents the chemical potential at the A-phase side of the interface. $G^{c o h}$ means the elastic energy required to maintain coherence at the interface, defined by $\left(u_{i j}^{M}-u_{i j}^{P}\right) \sigma_{i j}^{M}$, where $u_{i j}^{A}$ and $\sigma_{i j}^{A}$ are elastic strain and stress tensors at the A-phase side of the interface, respectively [16]. $G^{\text {trans }}$ represents the difference in the elastic strain energies of the precipitate and the matrix, as defined by $1 / 2 \sigma_{i j}^{P}\left(u_{i j}^{P}-e^{T} \delta_{i j}\right)-1 / 2 \sigma_{i j}^{M} u_{i j}^{M}[16]$. The detailed derivation of Eq. (12) will emerge elsewhere [12].

Numerical simulations on a discrete lattice were performed in two-dimensions. Euler's method was used for the integration in time. For all simulations presented 
here, the mesh size $\triangle x=1.0 \times 10^{-8} \mathrm{~m}$, the time step $\triangle t=0.0125$, and $\varepsilon=3.52 \times 10^{-5}$. This choice of $\triangle x$ and $\varepsilon$ guarantees that the interface is resolved by at least five points and the interfacial energy is $0.0243 \mathrm{~J} / \mathrm{m}^{2}$. We assume that the diffusivities of two phases are the same and $1 \times 10^{-15} \mathrm{~m}^{2} / \mathrm{s}$. Quadratic functions were used for the free energy functions of both phases for simplicity, that is, $f^{P}=\Delta^{P}\left(c^{P}-c_{e}^{P}\right)^{2} / V_{m}$ and $f^{M}=\Delta^{M}\left(c^{M}-c_{e}^{M}\right)^{2} / V_{m}$, where $\Delta^{P}$ and $\Delta^{M}$ were 10000 and 2000, respectively, $V_{m}$ is molar volume and was set at $7.0 \times 10^{-6} \mathrm{~m}^{3} / \mathrm{mol}$, and $c_{e}^{P}$ and $c_{e}^{M}$ are the equilibrium concentrations without elastic field and were set at 0.2 and 0.4 , respectively. The elastic constants of two phases (in units of $10^{11} \mathrm{erg} / \mathrm{cm}^{3}$ ) are $C_{11}^{P}=1.67, C_{12}^{P}=1.07, C_{44}^{P}=0.99, C_{11}^{M}=1.12$, $C_{12}^{M}=0.63$, and $C_{44}^{M}=0.57$, respectively; for the Nibased superalloy [5]. The misfit strain between the precipitate and the matrix is dilatational and assumed to be $0.8 \%$. The displacement field was calculated to the third order from Eq. (8) because the inhomogeneities of the elastic constants were very large. Periodic boundary conditions were employed in all directions.
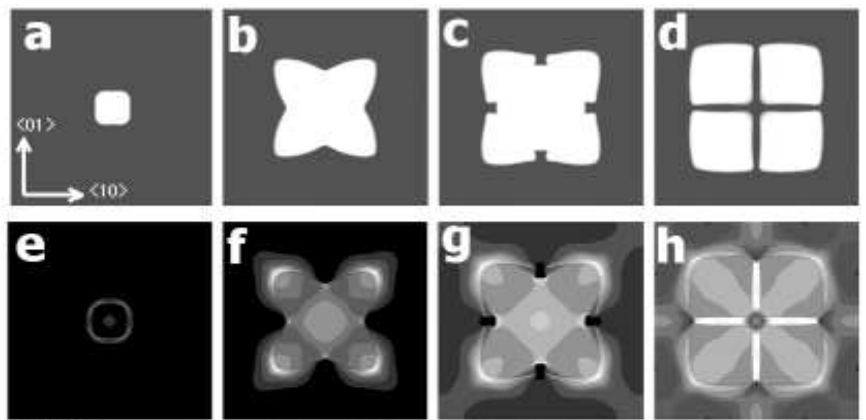

$f^{e l}(J)$

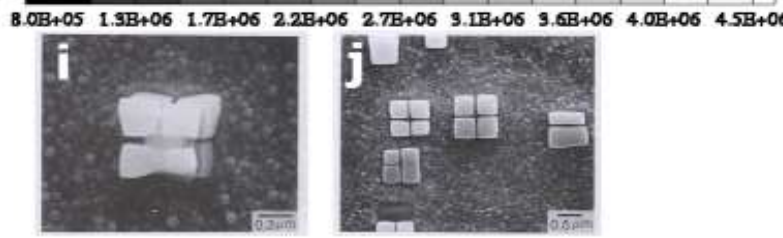

FIG. 1: Sequential evolutions of the precipitate (the top row) and the corresponding misfit elastic energy density distributions(the second row): from the left, $t=3.125,31.25,62.5$ and $150 \mathrm{~s}$. The lowest row shows splitting and split $\gamma /$-precipitates in the Ni-based superalloy system 3]. In the calculation, $M=5.0 \times 10^{-7}$. The total size of the calculation domain is $2.56 \mu \mathrm{m} \times 2.56 \mu \mathrm{m}$.

Figure 1 shows the calculated splitting behavior of the precipitate and the corresponding elastic energy density distribution at various times. The morphological instability of the interface due to the diffusion field and anisotropy of the elastic stiffness is evident and this interface instability leads to particle splitting. Despite the simplicity of the two-dimensional calculation, the splitting pattern is in good agreement with the experimental results (see Fig. 11 and j). The scenario of the splitting is as follows: initially the precipitate grows in a circular shape due to the isotropic interface energy, and then transforms to cuboidal shape above a critical size, because the misfit strain energy proportional to its volume dominates the interfacial energy in proportion to the interfacial area. Subsequently, the growth rate in the $\langle 11\rangle$ direction is enhanced due to a high chemical driving force while the migration rate in the $\langle 10\rangle$ direction is suppressed due to enriched solute and a corresponding low chemical driving force. Therefore, the growing shape transforms to a concave shape. In the concave growth regime, the interface in the $<10\rangle$ direction has a negative curvature, and so, the elastic strain energy concentrates around the concave edge . This concentrated elastic energy drives the dissolution of the precipitate, and then a groove forms and penetrates the precipitate (see Fig. 1k). This situation resembles AsaroTiller-Grinfeld instability, in which elastic media under the nonhydrostatic stress show the morphological instability [1]. Through various numerical simulations, we confirmed that the splitting occurs only in the concave growth condition and that it is induced by the interface instability. This is in good agreement with the experimental observations [1, 2, 3].
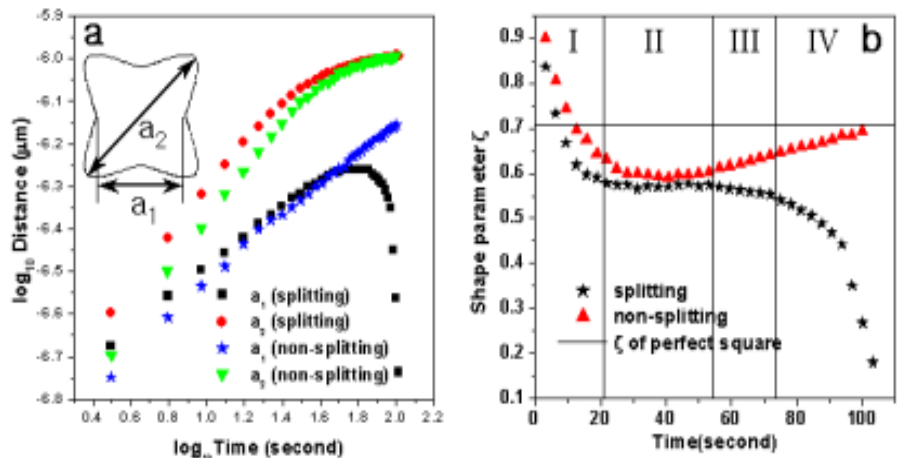

FIG. 2: Time variations of $<10>$ length $\left(a_{1}\right)$ and $<11>$ length $\left(a_{2}\right)$ of the precipitate (a) and the corresponding ratio of $a_{1}$ to $a_{2}$ (b) which is defined as the shape parameter $\zeta$. The inset shows the definitions of $a_{1}$ and $a_{2}$. In the case of splitting, $M=5.0 \times 10^{-7}$ and in non-splitting, $M=3.0 \times$ $10^{-7}$.

Figure 2 shows the sequential variations of the $\langle 10\rangle$ length $\left(a_{1}\right)$ and $<11>$ length $\left(a_{2}\right)$ of the precipitate and the ratio of the two lengths which is called a shape parameter $(\zeta)$ hereafter. There are four different growth modes (see Fig. 2 b). In the first growth mode the growth rates of $a_{1}$ and $a_{2}$ are proportional to $t^{0.35 \pm 0.005}$ and $t^{0.55 \pm 0.01}$, respectively and hence transition from circular through cuboidal to concave shapes occurs (for a perfect square $\zeta=0.7)$. The second regime can be called the shapeconserving mode because the shape parameter remains constant in this period. The growth rates of $a_{1}$ and $a_{2}$ 
have the same dependence $t^{0.31 \pm 0.01}$ on time. The growth of $a_{1}$ stops upon entering the third growth mode while $a_{2}$ continues to grow and the shape parameter starts to decrease again. In this regime the grooves are generated on the four interfaces of the $\langle 10\rangle$ direction (see Fig. (1) and concavity around the grooves starts to relax because the elastic field around the grooves is relieved by local concentration of the stress field on the edges of the grooves. One can see this by comparing Fig. 10 with Fig. 11. Once the elastic energy concentrated on the tips dominates the chemical driving force for precipitate growth which is reduced due to the buildup of solute around the grooves, the grooves penetrate into the precipitate and the fourth growth stage begins. Note that the particle in the non-splitting case also shows concave growth, but then returns to the cuboidal shape with time (see the variations of the filled triangles in Fig. 2b).
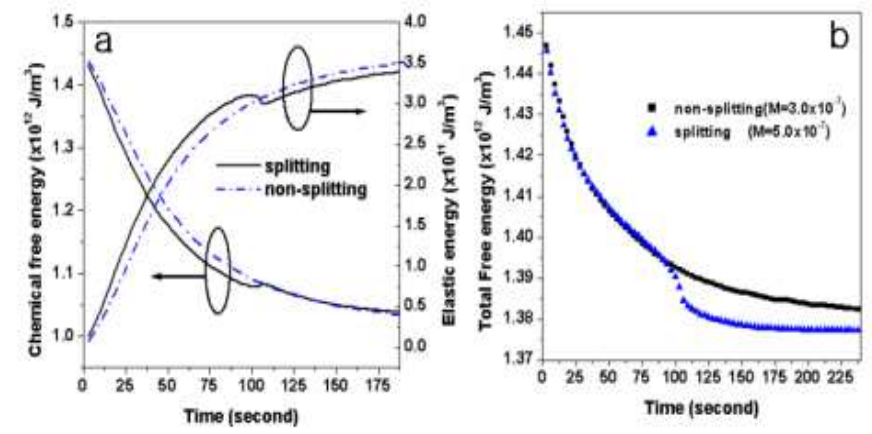

FIG. 3: Time variations of the total free energy (Eq. 2], elastic energy (a), and the total free energy, except the elastic energy (b). The phase field mobilities are $5.0 \times 10^{-7}$ for the splitting case and $3.0 \times 10^{-7}$ for the non-splitting case, respectively.

Fig. 3] shows the variations of the total free energy [Eq. (2)], elastic energy, and the total free energy excluding the elastic energy, with the evolution of the second particle. Here, the third free energy term mentioned above is called the chemical free energy although it includes the interfacial energy (i.e. gradient energy and double well potential energy). During the evolution of the particle, the total free energy and the chemical free energy decrease while the strain energy increases due to misfitting particle growth. When the splitting has been almost completed, the chemical free energy jumps due to the increased interfacial area, but the total strain energy reduces by interface grooving, which actually resembles the ATG instability [11]. As the decrease of strain energy overwhelms the increased interfacial free energy, the total free energy decreases and the splitting process advances. As shown in Fig. B $\mathrm{b}$, the configuration of four split pieces is a more stable state than non-split single particle. Hence the non-split large particle is consid- ered as a meta-stable phase and the activation barrier is the interfacial free energy corresponding to the increased interfacial area. Considering these points, the splitting phenomenon is kinetically rather than energetically driven.

In this work, it has been shown through phase field study that the interaction between the elastic field condensed at the concave region and solute enrichment causes the particle splitting phenomenon. The splitting is mediated by interface instability, which differs from previous studies that splitting occurs through the nucleation of the matrix phase at the center of the precipitate [5, 7] or that it is caused by energy considerations [6, 10]. In particular, the sequential splitting behavior realized by our simulation shows an excellent agreement with previous experimental studies [2, 3].

* Electronic address: dream@plaza1.snu.ac.kr

[1] A. Maheshwari and A.J. Ardell, Phys. Rev. Lett. 70, 2305 (1993); A.J. Ardell and R.B. Nicholson, Acta metall. 14, 1295 (1966).

[2] T. Miyazaki, K. Hakamura, and H. Mori, J. Mater. Sci. 14, 1827 (1979); M. Doi, T. Miyazaki, and T. Wakatsuki, Mater. Sci. Eng. 74, 139 (1985); H.A. Calderon, G. Kostorz, Y.Y. Qu, H.J. Dorantes, J.J. Cruz, and J.G. Cabanas-Moreno, Mat. Sci. Eng. A 238, 13 (1997).

[3] Y.S. Yoo, D.Y. Yoon, and M.F. Henry, Metals and Mater. 1, 47 (1995); M. Doi, T. Miyazaki, and T. Wakatsuki, Mater. Sci. Eng. 67, 247 (1984).

[4] P. Fratzl, O. Penrose, and J. Lebowitz, J. Stat. Phys. 95, 1429 (1999) and references therein.

[5] T. Miyazaki, H. Imamura, and T. Kozakai, Mater. Sci. Eng. 54, 9 (1982).

[6] A.G. Khachaturyan, S.V. Semenovskaya, and J.W. Jr Morris, Acta metall. 36, 1563 (1988).

[7] Y. Wang, L.-Q. Chen, and A.G. Khachaturyan, Acta metall. 41, 279 (1993).

[8] J.D. Zhang, D.Y. Li, and L.-Q. Chen, in Phase transformations and systems driven far from equilibrium, MRS, 481, 243 (1998).

[9] J.K. Lee, Int. Mater. Rev. 42, 221 (1997); J.K. Lee, Theor. Appl. Frac. Mech. 33, 207 (2000).

[10] M. Doi, Mater. Trans. JIM 33, 637 (1992).

[11] R.J. Asaro and W.A. Tiller, Metall. Trans. 3, 1789 (1972); M.A. Grinfeld, Sov. Phys. Dokl. 31, 831 (1986); D.J. Srolovitz, Acta metall. 37, 621 (1989).

[12] D.-H. Yeon, P.-R. Cha, M. Grant, and J.-K. Yoon, submitted to Phys. Rev. B.

[13] S.G. Kim, W.T. Kim, and T. Suzuki, Phys. Rev. E 60, 7186 (1999); P.-R. Cha, D.-H. Yeon, and J.-K. Yoon, Acta mater. 49, 3295 (2001).

[14] P.H. Leo, J.S. Lowengrub, and H.J. Jou, Acta mater. 46, 2113 (1998).

[15] S.Y. Hu and L.Q. Chen, Acta mater. 49, 1879 (2001).

[16] P.H. Leo and R.F. Sekerka, Acta metall. 37, 3139 (1989); P.H. Leo and R.F. Sekerka, Acta metall. 37, 3119 (1989). 